\title{
GAUSSIAN TRIVIAL RING EXTENSIONS AND FQP-RINGS
}

\author{
FRANÇOIS COUCHOT
}

\begin{abstract}
Let $A$ be a commutative ring and $E$ a non-zero $A$-module. Necessary and sufficient conditions are given for the trivial ring extension $R$ of $A$ by $E$ to be either arithmetical or Gaussian. The possibility for $R$ to be Bézout is also studied, but a response is only given in the case where $\operatorname{pSpec}(A)$ (a quotient space of $\operatorname{Spec}(A)$ ) is totally disconnected. Trivial ring extensions which are fqp-rings are characterized only in the local case. To get a general result we intoduce the class of fqf-rings satisfying a weaker property than fqpring. Moreover, it is proven that the finitistic weak dimension of a fqf-ring is 0,1 or 2 and its global weak dimension is 0,1 or $\infty$.
\end{abstract}

Trivial ring extensions are often used to give either examples or counterexamples of rings. One of the most famous is the chain ring $R$ which is not factor of a valuation domain (see [ $[$, X.6] and [7, Theorem 3.5]). This ring $R$ is the trivial ring extension of a valuation domain $D$ by a non-standard uniserial divisible $D$-module. This example gives a negative answer to a question posed by Kaplansky.

In [14, 15 and 11 there are many results on trivial ring extensions and many examples of such rings. In particular, necessary and sufficient conditions are given for the trivial ring extension of a ring $A$ by an $A$-module $E$ to be either arithmetical or Gaussian in the following cases: either $A$ is a domain and $K$ is its quotient field, or $A$ is local and $K$ is its residue field, and $E$ is a $K$-vector space.

In our paper more general results are shown. For instance the trivial ring extension $R$ of a ring $A$ by a non-zero $A$-module $E$ is a chain ring if and only if $A$ is a valuation domain and $E$ a divisible module, and $R$ is Gaussian if and only if $A$ is Gaussian and $E$ verifies $a E=a^{2} E$ for each $a \in A$. Complete characterizations of arithmetical trivial ring extensions are given too. But Bezout trivial ring extensions of a ring $A$ are characterized only in the case where $\operatorname{pSpec}(A)$ (a quotient space of $\operatorname{Spec}(A))$ is totally disconnected.

We also study trivial ring extensions which are fqp-rings. The class of fqprings was introduced in 1 by Abuhlail, Jarrar and Kabbaj. We get a complete characterization of trivial ring extensions which are fqp-ring only in the local case. Each fqp-ring is locally fqp and the converse holds if it is coherent, but this is not generally true. We introduce the class of fqf-rings which satisfy the condition "each finitely generated ideal is flat modulo its annihilator", and it is exactly the class of locally fqp-rings. So, trivial fqf-ring extensions are completely characterized. This new class of rings contains strictly the class of fqp-rings.

In [6] it is proven that each arithmetical ring has a finitistic weak dimension equal to 0,1 or 2 . We show that any fqf-ring satisfy this property too, and its global weak dimansion is 0,1 or $\infty$ as it is shown for fqp-rings in [1].

2010 Mathematics Subject Classification. 13F30, 13C11, $13 \mathrm{E} 05$.

Key words and phrases. chain ring, Gaussian ring, arithmetical ring, Bézout ring. 
All rings in this paper are associative and commutative with unity, and all modules are unital. We denote respectively $\operatorname{Spec}(A), \operatorname{Max}(A)$ and $\operatorname{Min}(A)$, the space of prime ideals, maximal ideals and minimal prime ideals of $A$, with the Zariski topology. If $I$ a subset of $R$, then we denote

$$
V(I)=\{P \in \operatorname{Spec}(A) \mid I \subseteq P\} \quad \text { and } \quad D(I)=\operatorname{Spec}(A) \backslash V(I) .
$$

Let $A$ be a ring and $E$ an $A$-module. The trivial ring extension of $A$ by $E$ (also called the idealization of $E$ over $A$ ) is the $\operatorname{ring} R:=A \propto E$ whose underlying group is $A \times E$ with multiplication given by $(a, e)\left(a^{\prime}, e^{\prime}\right)=\left(a a^{\prime}, a e^{\prime}+a^{\prime} e\right)$.

\section{Arithmetical RINGS}

An $R$-module $M$ is said to be uniserial if its set of submodules is totally ordered by inclusion and $R$ is a chain ring $]^{1}$ if it is uniserial as $R$-module. Recall that a chain ring $R$ is said to be Archimedean if its maximal ideal is the sole non-zero prime ideal.

Proposition 1.1. Let $A$ be a ring, $E$ a non-zero $A$-module and $R=A \propto E$ the trivial ring extension of $A$ by $E$. The following two conditions are equivalent:

(1) $R$ is a chain ring;

(2) $A$ is a valuation domain and $E$ is a uniserial divisible module.

Proof. It is well known that (2) $\Rightarrow(1)$ (for instance, see [7, Example I.1.9]). Conversely, $A$ is a chain ring because it is a factor of $R$, and $E$ is uniserial because it is isomorphic to an ideal of $R$. Let $0 \neq a \in A$ and $x \in E$. It is obvious that $(0, x) \in R(a, 0)$. So, $(0, x)=(a, 0)(b, y)$ for some $y \in E$ and $b \in A$, whence $x=a y$. Hence $E=a E$. Let $a, b$ be non-zero elements of $A$. Then $E=a E=a b E$. We deduce that $a b \neq 0$. Hence $A$ is a domain and $E$ is divisible.

Let $E$ be a module over a ring $R$. We say that $E$ has a distributive lattice of submodules if $E$ satisfies one of the following two equivalent conditions:

- $(M+N) \cap P=(M \cap P)+(N \cap P)$ for any submodules $M, N, P$ of $E$;

- $(M \cap N)+P=(M+P) \cap(N+P)$ for any submodules $M, N, P$ of $E$.

The following proposition can be proved as [13, Theorem 1].

Proposition 1.2. Let $E$ be a module over a ring $R$. The following conditions are equivalent:

(1) E has a distributive lattice of submodules;

(2) $E_{P}$ is a uniserial module for each maximal ideal $P$ of $R$.

A ring (respectively domain) $R$ is said to be arithmetical (respectively Prüfer) if its lattice of ideals is distributive. An $R$-module $E$ is FP-injective if, for each finitely presented $R$-module $F$, $\operatorname{Ext}_{R}^{1}(F, E)=0$. When $R$ is a Prüfer domain then a module is FP-injective if and only if it is divisible.

Lemma 1.3. Let $A$ be a ring, $E$ a non-zero $A$-module and $R=A \propto E$ the trivial ring extension of $A$ by $E$. Let $S$ be a multiplicative subset of $R$ and $S^{\prime}$ the image of $S$ by the map $R \rightarrow A$ defined by $(a, x) \mapsto a$. Then $S^{-1} R=S^{\prime-1} A \propto S^{\prime-1} E$.

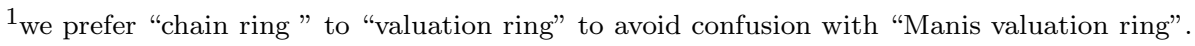


Proof. If $(a, x) \in R$ and $(s, y) \in S$ then it is easy to check that

$$
\frac{(a, x)}{(s, y)}=\left(\frac{a}{s}, \frac{s x-a y}{s^{2}}\right)=\frac{(s a, s x-a y)}{s^{2}}=\frac{(a, x)(s,-y)}{s^{2}} .
$$

For any module $E$ over $\operatorname{ring} R$ we define $\operatorname{Supp}(E)$ as:

$$
\operatorname{Supp}(E)=\left\{P \mid P \text { prime ideal such that } E_{P} \neq 0\right\} .
$$

An $R$-module $E$ is locally $\mathbf{F P}$-injective if for each maximal ideal $P, E_{P}$ is FPinjective.

From Propositions 1.1 and 1.2 and Lemma 1.3 we deduce the following corollary.

Corollary 1.4. Let $A$ be a ring, $E$ a non-zero $A$-module and $R=A \propto E$ the trivial ring extension of $A$ by $E$. The following two conditions are equivalent:

(1) $R$ is arithmetical;

(2) $A$ is arithmetical, $A_{P}$ is an integral domain for each $P \in \operatorname{Supp}(E)$, and $E$ is locally FP-injective and has a distributive lattice of submodules.

Remark 1.5. Let us observe that $E$ is a module over $A / N$ where $N$ is the nilradical of $A$ if $R$ is arithmetical.

Proof. Let $a \in N$ and $P$ be a maximal ideal of $A$. Either $P \in \operatorname{Supp}(E)$ and $a R_{P}=0$, or $E_{P}=0$. So, $a E=0$.

\section{Gaussian Rings}

Let $R$ be a ring. For a polynomial $f \in R[X]$, denote by $c(f)$ (the content of $f$ ) the ideal of $R$ generated by the coefficients of $f$. We say that $R$ is Gaussian if $c(f g)=c(f) c(g)$ for any two polynomials $f$ and $g$ in $R[X]$. By [19], a local ring $R$ is Gaussian if and only if, for any ideal $I$ generated by two elements $a, b$, in $R$, the following two properties hold:

(1) $I^{2}$ is generated by $a^{2}$ or $b^{2}$;

(2) if $I^{2}$ is generated by $a^{2}$ and $a b=0$, then $b^{2}=0$.

Proposition 2.1. Let $A$ be a local ring, $E$ a non-zero $A$-module and $R=A \propto E$ the trivial ring extension of $A$ by $E$. The following two conditions are equivalent:

(1) $R$ is Gaussian;

(2) $A$ is Gaussian and $a E=a^{2} E$ for each $a \in A$.

Proof. (1) $\Rightarrow(2)$. As factor of $R, A$ is Gaussian. Let $0 \neq a \in A$ and $x \in E$. First assume $a^{2} \neq 0$. Then $(a, 0)^{2} \neq 0$ and $(0, x)^{2}=0$. So, $(a, 0)(0, x)=(0, a x)=$ $(0, y)\left(a^{2}, 0\right)=\left(0, a^{2} y\right)$ for some $y \in E$. We get $a^{2} y=a x$. Now assume $a^{2}=0$. In this case $(a, 0)^{2}=0$. It follows that $(a, 0)(0, x)=0$, whence $a x=0$. In the two cases $a E=a^{2} E$.

$(2) \Rightarrow(1)$. Let $r=(a, x)$ and $s=(b, y)$ two elements of $R$. Since $A$ is Gaussian we may assume that $a b=c a^{2}$ and $b^{2}=d a^{2}$ for some $c, d \in A$. We shall prove that $(R r+R s)^{2}=R r^{2}$ by showing there exist $z$ and $v$ in $E$ satisfying the following two equations:

$$
\begin{gathered}
(a, x)(b, y)=(c, z)(a, x)^{2} \\
(b, y)^{2}=(d, v)(a, x)^{2}
\end{gathered}
$$


For (11) we only need to show that $a^{2} z=a y+b x-2 a c x$. Since $b E=b^{2} E$ then $b x=b^{2} m=d a^{2} m$ for some $m \in E$. So, $a y+b x-2 a c x=a(y+a d m-2 c x)$. Now we use the equality $a E=a^{2} E$ to conclude.

For (21) we only need to show that $a^{2} v=2 b y-2 a d x$. Since $b E=b^{2} E$ then $b y=b^{2} n=d a^{2} n$ for some $n \in E$. So, $2 b y-2 a d x=a(2 a d n-2 d x)$. Now we use the equality $a E=a^{2} E$ to conclude.

If $r s=0$ then $a b=0$ and $b^{2}=0$. So, $s^{2}=(0,2 b y)$. But $b E=b^{2} E=0$, whence $b y=0$ and $s^{2}=0$.

Corollary 2.2. Let $A$ be a ring, $E$ a non-zero $A$-module and $R=A \propto E$ the trivial ring extension of $A$ by $E$. The following two conditions are equivalent:

(1) $R$ is Gaussian;

(2) $A$ is Gaussian and $a E=a^{2} E$ for each $a \in A$.

Proof. We deduce this corollary from Proposition 2.1 and from the fact that a ring $A$ is Gaussian if and only $A_{P}$ is Gaussian for each maximal ideal $P$ and that $a E=a^{2} E$ if and only if $a E_{P}=a^{2} E_{P}$ for each maximal ideal $P$.

Remark 2.3. Let $A$ be a Gaussian ring. Let $E$ be an $A$-module such that $a E=$ $a^{2} E$ for each $a \in A$. If $a \in N$, where $N$ is the nilradical of $A$, it is easy to see that $a E=0$. So, $E$ is a module over $A / N$ which is arithmetical by [17, Corollary 7].

Proposition 2.4. Let $A$ be an integral domain and $E$ an $A$-module such that $a E=a^{2} E$ for each $a \in A$. Then:

(1) $E$ is divisible if it is torsion-free;

(2) when $A$ is local, $E$ is semisimple if it is finitely generated;

(3) if $A$ is an Archimedean valuation domain, then $E$ is the extension of a divisible module by a semisimple module.

Proof. (1). Let $0 \neq a \in A$ and $x \in E$. There exists $y \in E$ such that $a x=a^{2} y$. Since $E$ is torsion-free we get $x=a y$.

(2). Let $P$ be the maximal ideal of $A$ and $a \in P$. We have $a E=a(a E)$. By Nakayama Lemma $a E=0$. So, $E$ is an $A / P$-module.

(3). To do this we show that $P E$ is divisible. Let $x \in P E$ and $0 \neq s \in A$. Then $x=a y$ for some $a \in P$ and $y \in E$. It is easy to check that $\cap_{n \in \mathbb{N}} A a^{n}$ is a prime ideal. So, this intersection is 0 . There exists an integer $n$ such that $s \notin A a^{n}$. We get that $a E=s a E=a^{n+1} E$. Hence $x \in s a E \subseteq s P E$.

Example 2.5. Let $A$ be a valuation domain, $\Lambda$ an index set and $\left(L_{\lambda}\right)_{\lambda \in \Lambda}$ a family of prime ideals. For each $\lambda \in \Lambda$ let $E_{\lambda}$ be a non-zero divisible $A / L_{\lambda}$-module. Let $E_{1}=\oplus_{\lambda \in \Lambda} E_{\lambda}$ and $E_{2}=\prod_{\lambda \in \Lambda} E_{\lambda}$. Then $a E_{i}=a^{2} E_{i}$ for each $a \in A$ and for $i=1,2$.

Proof. Let $0 \neq a \in A$ and $x=\left(x_{\lambda}\right)_{\lambda \in \Lambda} \in E_{i}$ where $i=1$, 2. If $a x_{\lambda}=0$ then $a x_{\lambda}=a^{2} y_{\lambda}$ with $y_{\lambda}=0$. If $a x_{\lambda} \neq 0$ then $a \notin L_{\lambda}$. Since $E_{\lambda}$ is divisible over $A / L_{\lambda}$ there exists $y_{\lambda} \in E_{\lambda}$ such that $a x_{\lambda}=a^{2} y_{\lambda}$. So, $a x=a^{2} y$ with $y=\left(y_{\lambda}\right)_{\lambda \in \Lambda}$.

Let $A$ be a valuation domain. A non-zero prime ideal $L$ that is not the union of prime ideals properly contained in it is called branched.

Proposition 2.6. Let $A$ be a valuation domain. Assume that each non-zero prime ideal is branched. For any $A$-module $E$ the following conditions are equivalent:

(1) $a E=a^{2} E$ for each $a \in A$; 
(2) for each prime ideal $L, L E / L^{\prime} E$ is an $A / L^{\prime}$-module divisible, where $L^{\prime}$ is the union of all prime ideals properly contained in $L$.

Proof. (1) $\Rightarrow(2)$. Let $s \in L \backslash L^{\prime}$ and $x \in L E$. Then $x=a y$ for some $a \in L$ and $y \in E$. We may assume that $a \notin L^{\prime}$. So, $L^{\prime}=\cap_{n \in \mathbb{N}} A a^{n}$ and there exists $n \in \mathbb{N}$ such that $s \notin A a^{n}$. Whence $a E=a^{n+1} E \subseteq s a E \subseteq a E$. Hence $x \in s L E$.

$(2) \Rightarrow(1)$. Let $0 \neq a$ a non-unit of $A$. Let $L$ be the prime ideal which is the intersection of all prime ideals containing $a$. Then $a \notin L^{\prime}$. Let $x=a y \in a E$. Since $L E / L^{\prime} E$ is divisible over $A / L^{\prime}$, then $x \in a^{2} E+L^{\prime} E \subseteq a^{2} E$.

A chain ring is said to be strongly discrete if it contains no idempotent prime ideal. Each non-zero prime ideal of a strongly discrete valuation domain is branched.

\section{BÉzOUT RINGS}

A ring is a Bézout ring if every finitely generated ideal is principal. A ring $R$ is Hermite if $R$ satisfies the following property : for every $(a, b) \in R^{2}$, there exist $d, a^{\prime}, b^{\prime}$ in $R$ such that $a=d a^{\prime}, b=d b^{\prime}$ and $R a^{\prime}+R b^{\prime}=R$. We say that $R$ is an elementary divisor ring if for every matrix $A$, with entries in $R$, there exist a diagonal matrix $D$ and invertible matrices $P$ and $Q$, with entries in $R$, such that $P A Q=D$. Then we have the following implications:

elementary divisor ring $\Rightarrow$ Hermite ring $\Rightarrow$ Bézout ring $\Rightarrow$ arithmetical ring;

but these implications are not reversible: see [9] or [4.

Proposition 3.1. Let $A$ be a ring and $N$ its nilradical. Assume that $N$ is prime. Let $E$ be a non-zero $A$-module and $R=A \propto E$ the trivial ring extension of $A$ by

$E$. The following three conditions are equivalent:

(1) $R$ is Hermite;

(2) $R$ is Bézout;

(3) A is Bézout, $A_{P}$ is a domain for each $P \in \operatorname{Supp}(E), E$ is FP-injective and all its finitely generated submodules are cyclic.

Proof. (1) $\Leftrightarrow(2)$. It is well known that each Hermite ring is Bézout. Since $R$ contains a unique minimal prime ideal, then the converse holds by [12, Theorem 2].

$(2) \Rightarrow(3)$. By Corollary $1.4 E$ is FP-injective and $A_{P}$ is a domain for each $P \in \operatorname{Supp}(E)$. As factor of $R A$ is Bézout, and each finitely generated submodule of $E$ is cyclic because $E$ is isomorphic to an ideal of $R$.

$(3) \Rightarrow(2)$. Let $a \in N$ and $x \in E$. For each $P \in \operatorname{Supp}(E) a A_{P}=0$ (since $A_{P}$ is a domain). It follows that $(0: a) \nsubseteq \subseteq P$ and consequently $\operatorname{Supp}(E) \subseteq D((0: a))$. For each $P \notin \operatorname{Supp}(E), A_{P} x=0$. It follows that $(0: x) \nsubseteq P$ and consequently $D((0: a)) \cup D((0: x))=\operatorname{Spec}(A)$. So, $(0: a)+(0: x)=A$, whence there exist $b \in(0: a)$ and $c \in(0: x)$ such that $b+c=1$. Then $a=c a, x=b x$ and it is easy to check that $R(a, 0)+R(0, x)=R(a, x)$. Let $(a, x)$ and $(b, y)$ be two elements of $R$. If $(a, b) \in N \times N$ then there exist $d \in A$ such that $A d=A a+A b$, and $z \in E$ such that $A x+A y=A z$. It follows that $R(a, x)+R(b, y)=R(d, z)$. Now assume that $(a, b) \notin N \times N$. There exist $d, a^{\prime}, b^{\prime}, s, t \in A$ such that $a=d a^{\prime}, b=d b^{\prime}$ and $s a+t b=d$. Let $z=s x+t y$. Since $E$ is divisible over $A / N$ and $d \notin N$, there exist $x^{\prime}, y^{\prime} \in E$ such that $x-a^{\prime} z=d x^{\prime}$ and $y-b^{\prime} z=d y^{\prime}$. Now it is easy to check that $(a, x)=(d, z)\left(a^{\prime}, x^{\prime}\right),(b, y)=(d, z)\left(b^{\prime}, y^{\prime}\right)$ and $(s, 0)(a, x)+(t, 0)(b, y)=(d, z)$.

If $R$ is a ring, we consider on $\operatorname{Spec}(R)$ the equivalence relation $\mathcal{R}$ defined by $L \mathcal{R} L^{\prime}$ if there exists a finite sequence of prime ideals $\left(L_{k}\right)_{1 \leq k \leq n}$ such that $L=L_{1}, L^{\prime}=L_{n}$ 
and $\forall k, 1 \leq k \leq(n-1)$, either $L_{k} \subseteq L_{k+1}$ or $L_{k} \supseteq L_{k+1}$. We denote by $\operatorname{pSpec}(R)$ the quotient space of $\operatorname{Spec}(R)$ modulo $\mathcal{R}$ and by $\lambda_{R}: \operatorname{Spec}(R) \rightarrow \operatorname{pSpec}(R)$ the natural map. The quasi-compactness of $\operatorname{Spec}(R)$ implies the one of $\operatorname{pSpec}(R)$, but generally $\operatorname{pSpec}(R)$ is not $T_{1}$ : see [16, Propositions 6.2 and 6.3]. A topological space is called totally disconnected if each of its connected components contains only one point. Every Hausdorff topological space $X$ with a base of clopen neighbourhoods is totally disconnected and the converse holds if $X$ is compact (see [11, Theorem 16.17]).

An ideal $I$ of a ring $A$ is pure if and only if $A / I$ is a flat $A$-module.

Theorem 3.2. Let $A$ be a ring, $E$ a non-zero $A$-module and $R=A \propto E$ the trivial ring extension of $A$ by $E$. Assume that $\operatorname{pSpec}(A)$ is totally disconnected. The following three conditions are equivalent:

(1) $R$ is Hermite;

(2) $R$ is Bézout;

(3) $A$ is is Bézout, $A_{P}$ is a domain for each $P \in \operatorname{Supp}(E)$, $E$ is locally FPinjective and all its finitely generated submodules are cyclic.

Proof. $(1) \Rightarrow(2)$ is well known and we show $(2) \Rightarrow(3)$ as in Proposition 3.1

$(3) \Rightarrow(1)$. By [5, Proposition 2.2] $\operatorname{pSpec}(A)$ is compact. Since $A=R / J$ where $J$ is contained in the nilradical of $R$ then $\operatorname{pSpec}(A)$ and $\operatorname{pSpec}(R)$ are homeomorphic. Let $x \in \operatorname{pSpec}(R), I(x)$ the pure ideal of $R$ for which $x=V(I(x))$ and $I^{\prime}=$ $I(x) / N I(x)$ (see [5, Lemma 2.5]). Thus $I^{\prime}$ is a pure ideal of $A$ and it is contained in only one minimal prime ideal. So, the nilradical of $A / I^{\prime}$ is prime. If $S=1+I(x)$ then $S^{-1} R=R / I(x)$. By using Lemma 1.3 we get that $R / I(x)=A / I^{\prime} \propto E / I^{\prime} E$. By Proposition 3.1 $R / I(x)$ is Bézout for each $x \in \operatorname{pSpec}(R)$. We conclude that $R$ is Hermite by [5, Theorem 3.1].

Recall that a ring $A$ is coherent (respectively semihereditary) if all its finitely generated ideals are finitely presented (respectively projective).

Corollary 3.3. Let $A$ be a coherent reduced ring, $E$ a non-zero A-module and $R=A \propto E$ the trivial ring extension of $A$ by $E$. The following three conditions are equivalent:

(1) $R$ is Hermite;

(2) $R$ is Bézout;

(3) $A$ is is Bézout, E is FP-injective and all its finitely generated submodules are cyclic.

Proof. Since $A$ is Bézout, reduced and coherent then $A$ is semihereditary. By [18, Proposition 10] $\operatorname{Min}(A)$ is compact, and by [5. Proposition 2.2] $\operatorname{pSpec}(A)$ is homeomorphic to $\operatorname{Min}(A)$, and consequently it is totally disconnected. Since $A$ is coherent, each FP-injective module is locally FP-injective. We conclude by Theorem 3.2

Corollary 3.4. Let $A$ be a ring, $E$ a non-zero $A$-module and $R=A \propto E$ the trivial ring extension of $A$ by $E$. Assume that $\operatorname{pSpec}(A)$ is totally disconnected. The following two conditions are equivalent:

(1) $R$ is an elementary divisor ring;

(2) $A$ is an elementary divisor ring, $A_{P}$ is a domain for each $P \in \operatorname{Supp}(E), E$ is locally FP-injective and all its finitely generated submodules are cyclic. 
Proof. It is a consequence of Theorem 3.2 and the following. By [10, Theorem 6] a ring $R$ is an elementary divisor ring if and only if $R$ is Hermite and for any $a, b, c \in R$ such that $R a+R b+R c=R$ there exist $p, q \in R$ such that $R p a+R(p b+q c)=R$. By [12, Theorem 3] a Hermite ring $R$ is an elementary divisor ring if and only if so is $R / N$, where $N$ is the nilradical of $R$.

Corollary 3.5. Let $A$ be a coherent reduced Bézout ring, E a non-zero FP-injective $A$-module. Assume that each finitely generated submodule of $E$ is cyclic. Then, for any $x, y \in E$ there exist $z \in E$ and an invertible $2 \times 2$ matrix $B$ with coefficients in A such that $\left(\begin{array}{l}z \\ 0\end{array}\right)=B\left(\begin{array}{l}x \\ y\end{array}\right)$.

Proof. Let $R=A \propto E$. Let $x, y \in A$. By Corollary $3.3 R$ is Hermite. So, there exist $r \in R$ and an invertible $2 \times 2$ matrix $C$ with entries in $R$ such that $\left(\begin{array}{l}r \\ 0\end{array}\right)=C\left(\begin{array}{l}(0, x) \\ (0, y)\end{array}\right)$. It is obvious that $r=(0, z)$ for some $z \in E$. From $C$ we deduce an invertible $2 \times 2$ matrix $B$ with entries in $A$ satisfying $\left(\begin{array}{l}z \\ 0\end{array}\right)=B\left(\begin{array}{l}x \\ y\end{array}\right)$.

Contrary to the two first sections we do not get general results, even in the case where $A$ is reduced. In [20] there are two examples of reduced Bézout rings which are not semihereditary. For the first example $A$ ([20, Example 1.3b]) $\operatorname{pSpec}(A)$ is totally disconnected, so, if $E$ is an $A$-module satisfying the conditions of Theorem 3.2 then $A \propto E$ is Bézout. But for the second example $A$ ([20, Example 6.2]), $\operatorname{pSpec}(A)$ is connected and infinite, so, we do not know if $A$ admits Bézout proper trivial ring extensions.

\section{FQP-RINGS AND FQF-RINGS}

Let $A$ be a ring, $M$ an $A$-module. An $A$-module $\mathrm{V}$ is $M$-projective if the natural homomorphism $\operatorname{Hom}_{A}(V, M) \rightarrow \operatorname{Hom}_{A}(V, M / X)$ is surjective for every submodule $X$ of $M$. We say that $V$ is quasi-projective if $V$ is $V$-projective. A ring $A$ is said to be an fqp-ring if every finitely generated ideal of $A$ is quasi-projective.

Theorem 4.1. Let $A$ a local ring and $N$ its nilradical. Then $A$ is an fqp-ring if and only if either $A$ is a chain ring or $A / N$ is a valuation domain and $N$ is a divisible torsionfree $A / N$-module.

Proof. Assume that $A$ is an fqp-ring but not a chain ring. By [1, Lemmas 3.12 and 4.5], $N^{2}=0$ and every zero-divisor belongs to $N$. So, $N$ is prime. From [1, Lemma $3.8]$ it follows that any two elements of $A$ which are not in $N$ are comparable. This implies that $A / N$ is a valuation domain. Let $a \notin N$ and $b \in N$. By using again [1, Lemma 3.8] we get that $b \in A a$. Hence $N$ is divisible over $A / N$ and it is torsionfree since each element in $A \backslash N$ is regular.

Conversely, it is easy to see that each chain ring is fqp. So, we may assume that $A$ is not a chain ring. Let $I$ a finitely generated ideal of $A$. If $I \subseteq N$ then $I$ is a free module over $A / N$. Consequently $I$ is quasi-projective. Now, suppose that $I \nsubseteq N$. Thus $(I+N) / N$ is principal, and from the fact that $N$ is divisible over $A / N$ we deduce that $I$ is principal too. Hence $I$ is quasi-projective.

Theorem 4.2. Let $A$ be a local ring, $N$ its nilradical, $E$ a non-zero A-module and $R=A \propto E$ the trivial ring extension of $A$ by $E$. Then $R$ is an fqp-ring if and only if $A$ is an fqp-ring and one of the following conditions holds: 
(1) $A$ is a valuation domain and $E$ is divisible and uniserial;

(2) $E$ is divisible and torsionfree over $A / N$, each zero-divisor of $A$ belongs to $N$ and $N^{2}=0$.

Proof. First assume that $R$ is fqp. By [1, Proposition 4.1] so is $A$. If $R$ is a chain ring we use Proposition 1.1. If not, since $R$ is Gaussian by [1, Theorem 3.2] then $E$ is an $A / N$-module by Remark 2.3 . Let $N^{\prime}$ be the nilradical of $R$. It is easy to see that $R / N^{\prime} \cong A / N$ and $N^{\prime} \cong N \oplus E$. By Theorem 4.1, $N^{\prime 2}=0, N^{2}=0$ and $E$ is divisible and torsionfree over $A / N$.

Conversely, condition (1) implies that $R$ is a chain ring. Now, assume that condition (2) holds. As in the first part of the proof, $R / N^{\prime} \cong A / N, N^{\prime} \cong N \oplus E$ and $N^{\prime 2}=0$. So, $N^{\prime}$ is divisible and torsionfree over $R / N^{\prime}$. We conclude by Theorem 4.1 .

Corollary 4.3. Let $A$ be a valuation domain which is not a field, E a non-zero divisible $A$-module and $R=A \propto E$. Then:

(1) if $E$ is uniserial then $R$ is a chain ring;

(2) if $E$ is torsionfree but not uniserial then $R$ is an fqp-ring which is not arithmetical;

(3) if $E$ is neither torsionfree nor uniserial then $R$ is Gaussian but not an fqp-ring.

Proposition 4.4. Let $A$ be a coherent ring. Then $A$ is an fqp-ring if and only so is $A_{P}$ for each maximal ideal $P$ of $A$.

Proof. Let $I$ be a finitely generated ideal. Then $(\operatorname{End}(I))_{P} \cong \operatorname{End}\left(I_{P}\right)$ since $I$ is finitely presented. We use [1, Lemma 3.7] to conclude.

We say that a ring $A$ is a fqf-ring if each finitely generated ideal $I$ is flat modulo its annihilator, i.e $I$ is a flat $A /(0: I)$-module. It is obvious that every fqp-ring $A$ is a fqf-ring (by [1, Lemma 2.2] a finitely generated module is quasi-projective if and only if it is projective modulo its annihilator). By Proposition 4.4 the converse holds if $A$ is coherent. But we shall see that it is not generally true.

Corollary 4.5. Let $A$ be a ring, $N$ its nilradical, $E$ a non-zero $A$-module and $R=A \propto E$. Then the following conditions are equivalent:

(1) $R$ is a fqf-ring;

(2) $A$ is a fqf-ring and for each $P \in \operatorname{Supp}(E)$ either $N_{P}=0$ and $E_{P}$ is a uniserial divisible $A_{P}$-module or $N_{P}^{2}=0$ and $E_{P}$ and $N_{P}$ are divisible and torsionfree over $A_{P} / N_{P}$.

Proof. Over a local ring each finitely generated flat module is free. So, a local ring is a fqf-ring if and only if it is an fqp-ring (by [1, Lemma 2.2]). As a module is flat if and only if it is locally flat, then a ring $A$ is a fqf-ring if and only if so is $A_{P}$ for each maximal ideal $P$ of $A$. So, we use Theorem 4.2 to conclude.

Example 4.6. Let $A$ be a von Neumann regular ring which is not self-injective, $H$ the injective hull of $A, x \in H \backslash A, E=A+A x$ and $R=A \propto E$. Then $R$ is a fqf-ring which is not an fqp-ring.

Proof. Clearly $A$ is a reduced fqp-ring, and, for each maximal ideal $P, E_{P}$ is injective and torsionfree over the field $A_{P}$. By corollary $4.5 R$ is a fqf-ring. On the other hand $E$ is isomorphic to a finitely generated ideal $J$ of $R$. It is obvious that 
$E$ is a faithful $A$-module, so its annihilator as $R$-module is $J$. If $P \in \operatorname{Supp}(E / A)$ then $E_{P}$ is of rank 2 and if $P \notin \operatorname{Supp}(E / A)$ it is of rank one. But $(A: x)$ is an essential ideal of $A$ and consequently it is not generated by an idempotent. So, $\operatorname{Supp}(E / A)=V(\operatorname{ann}(E / A))=V((A: x))$ is not open . It follows that the map $\operatorname{Spec}(A) \rightarrow \mathbb{N}$ defined by $P \mapsto \operatorname{rank}_{A_{P}}\left(E_{P}\right)$ is not locally constant. Hence $E$ is not a projective $A$-module by [3, Théorème II.§5.2.1]. By [1, Lemma 2.2] it is not quasi-projective over $R$, whence $R$ is not an fqp-ring.

\section{Finitistic And GLOBAL WeAK DIMENSIONS OF FQF-RINGS}

Let $R$ be a ring. If $M$ is an $R$-module, we denote by w.d.( $M)$ its weak dimension. Recall that w.d. $(M) \leq n$ if $\operatorname{Tor}_{n+1}^{R}(M, N)=0$ for each $R$-module $N$. For any ring $R$, its global weak dimension w.gl.d $(R)$ is the supremum of w.d.( $M)$ where $M$ ranges over all (finitely presented cyclic) $R$-modules. Its finitistic weak dimension f.w.d. $(R)$ is the supremum of w.d. $(M)$ where $M$ ranges over all $R$ modules of finite weak dimension.

We shall extend [1, Theorem 3.11] and[6, Theorem 1] to fqf-rings by showing the following theorem:

Theorem 5.1. The global weak dimension of a fqf-ring is 0,1 or $\infty$ and its finitistic weak dimension is 0,1 or 2 .

Proof. The first assertion can be proven as [1, Theorem 3.11].

Let $R$ be a fqf-ring. It is well known that an $R$-module $M$ is flat if and only if so is $M_{P}$ for each $P \in \operatorname{Max} R$. So, we may assume that $R$ is local. By Theorem 4.2 either $R$ is a chain ring and we use [6, Theorem 2] or $R$ satisfies the assumptions of the following proposition and we conclude by using it.

Proposition 5.2. Let $R$ be a local ring and $N$ its nilradical. Assume that $R / N$ is a valuation domain, $N^{2}=0$ and $N$ is divisible and torsionfree over $R / N$. Then:

(1) if $N$ is the maximal ideal then f.w.d. $(R)=0$;

(2) if $N$ is not maximal then f.w.d. $(R)=1$.

Proof. (1). In this case $R$ is a primary ring. We conclude by 2, Theorems $\mathrm{P}$ and 6.3].

(2). Let $Q$ be the quotient ring of $R$. Since each element of $R$ which is not in $N$ is regular then $Q=R_{N}$ and since $N$ is divisible and torsionfree over $R / N$ then $N$ is a $Q$-module and it is the maximal ideal of $Q$. From (1) we deduce that f.w.d. $(Q)=0$.

Let $M$ be an $R$-module with w.d. $(M)<\infty$. Then we have w.d. $\left(M_{N}\right)<\infty$. So, $M_{N}$ is flat. Let $I$ be a finitely generated proper ideal. Then either $I \nsubseteq N$ and $I=R a$ for some $a \in R \backslash N$, or $I \subseteq N$ and $I \cong(R / N)^{n}$ for some integer $n \geq 1$.

In the first case we have w.d. $(R / I)=1$ since $a$ is a regular element, whence $\operatorname{Tor}_{2}^{R}(R / I, M)=0$. In the other case we have the following exact sequence:

$$
0 \rightarrow N F \rightarrow F \rightarrow I \rightarrow 0
$$

where $F$ is a free $R$-module of rank $n$. We deduce that the sequence

$$
0 \rightarrow N F_{N} \rightarrow F_{N} \rightarrow I_{N} \rightarrow 0 \text { is exact. }
$$


We have $\operatorname{Tor}_{1}^{Q}\left(I_{N}, M_{N}\right)=0$. Since $N$ is a $Q$-module, $N F \cong N F_{N}$. So, in the following commutative diagram

$$
\begin{array}{ccccc}
0 \rightarrow \operatorname{Tor}_{1}^{R}(I, M) & \rightarrow & N F \otimes_{R} M & \rightarrow & F \otimes_{R} M \\
& & \downarrow & & \downarrow \\
0 & \rightarrow & N F_{N} \otimes_{Q} M_{N} & \rightarrow & F_{N} \otimes_{Q} M_{N}
\end{array}
$$

the left vertical map is an isomorphism. Then $N F \otimes_{R} M \rightarrow F \otimes_{R} M$ is a monomorphism. We successively deduce that $\operatorname{Tor}_{1}^{R}(I, M)=0$ and $\operatorname{Tor}_{2}^{R}(R / I, M)=0$. Hence w.d. $(M) \leq 1$.

\section{REFERENCES}

[1] J. Abuhlail, V. Jarrar, and S. Kabbaj. Commutative rings in which every finitely generated ideal is quasi-projective. J. Pure Appl. Algebra, 215:2504-2511, (2011).

[2] H. Bass. Finitistic dimension and a homological generalization of semi-primary rings. Trans. Amer. Math. Soc., 95:466-488, (1960).

[3] N. Bourbaki. Algèbre commutative, Chapitres 1 et 2. Hermann, Paris, (1961).

[4] P. Carbonne. Anneaux de Bézout, Hermite, Kaplansky "universels". Canad. Math. Bull., 30(4):461-470, (1987).

[5] F. Couchot. Almost clean rings and arithmetical rings. In Commutative algebra and its applications, pages 135-154. Walter de Gruyter, (2009).

[6] F. Couchot. Finitistic weak dimension of commutative arithmetical rings. Arabian J. Math., 1(1):63-67, (2012).

[7] L. Fuchs and L. Salce. Modules over valuation domains, volume 97 of Lecture Notes in Pure an Appl. Math. Marcel Dekker, New York, (1985).

[8] L. Fuchs and L. Salce. Modules over Non-Noetherian Domains. Number 84 in Mathematical Surveys and Monographs. American Mathematical Society, Providence, (2001).

[9] L. Gillman and M. Henriksen. Rings of continuous functions in which every finitely ideal is principal. Trans. Amer. Math. Soc., 82:366-391, (1956).

[10] L. Gillman and M. Henriksen. Some remarks about elementary divisor rings. Trans. Amer. Math. Soc., 82:362-365, (1956).

[11] L. Gillman and M. Jerison. Rings of continuous functions. University Series in Higher Math. Van Nostrand, Princeton, N. J., (1960).

[12] M. Henriksen. Some remarks on elementary divisor rings II. Michigan Math.J., 3:159-163, (1955-56).

[13] C.U. Jensen. Arithmetical rings. Acta Math. Acad. Sci. Hungar., 17(1-2):115-123, (1966).

[14] S. Kabbaj and N. Mahdou. Trivial Extensions Defined by Coherent-like Conditions. Comm. Algebra, 32(10):3937-3953, (2004).

[15] M. Kabour and N. Mahdou. On Valuation Rings. Comm. Algebra, 39(1):176-183, (2011).

[16] D. Lazard. Disconnexités des spectres d'anneaux et des préschémas. Bull. Soc. Math. Fr., 95:95-108, (1967).

[17] T.G. Lucas. The Gaussian property for rings and polynomials. Houston J. Math., 34(1):1-18, (2008).

[18] Y. Quentel. Sur la compacité du spectre minimal d'un anneau. Bull. Soc. Math. France, 99:265-272, (1971).

[19] H. Tsang. Gauss's lemma. PhD thesis, University of Chicago, (1965).

[20] W.V. Vasconcelos. The rings of dimension two, volume 22 of Lecture Notes in pure and applied Mathematics. Marcel Dekker, (1976).

Université de Caen Basse-Normandie, CNRS UMR 6139 LMnO, F-14032 Caen, France

E-mail address: francois.couchot@unicaen.fr 